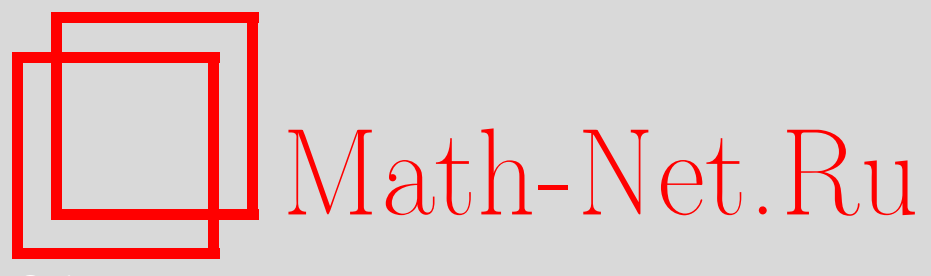

В. В. Волчков, Вит. В. Волчков, Поведение на бесконечности решений искаженного уравнения свертки, Изв. РАН. Сер. матем., 2012, том 76, выпуск 1, 85-100

DOI: https://doi.org/10.4213/im5405

Использование Общероссийского математического портала Math-Net.Ru подразумевает, что вы прочитали и согласны с пользовательским соглашением http://www . mathnet.ru/rus/agreement

Параметры загрузки:

IP : 107.22 .136 .117

26 апреля 2023 г., 02:18:47

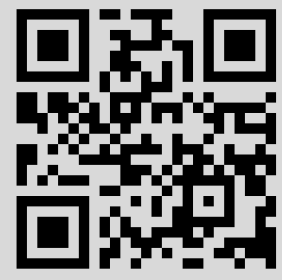


УДК 517.444

\author{
В. В. Волчков, Вит. В. Волчков
}

\title{
Поведение на бесконечности решений искаженного уравнения свертки
}

\begin{abstract}
Получены точные характеристики минимальной скорости роста на бесконечности ненулевых решений искаженного уравнения свертки в неограниченных областях из $\mathbb{C}^{n}$. В качестве приложений получены носящие окончательный характер уточнения теоремы о двух радиусах для искаженных сферических средних.

Библиография: 21 наименование.

Ключевые слова: группа Гейзенберга, искаженное уравнение свертки, теоремы о двух радиусах, вырожденные гипергеометрические функции.
\end{abstract}

\section{§ 1. Введение}

Пусть $\mathbb{C}^{n}$ - комплексное евклидово пространство размерности $n$ с евклидовой нормой $|\cdot|$ и эрмитовым скалярным произведением $\langle\cdot, \cdot\rangle_{\mathbb{C}}$. Обозначим

$$
\mathcal{B}_{r}(z)=\left\{w \in \mathbb{C}^{n}:|z-w|<r\right\}, \quad \overline{\mathcal{B}_{r}}(z)=\left\{w \in \mathbb{C}^{n}:|z-w| \leqslant r\right\}
$$

соответственно открытый и замкнутый шары в $\mathbb{C}^{n}$ с центром в точке $z$ и радиусом $r$. Положим $\mathcal{B}_{r}(0)=\mathcal{B}_{r}, \overline{\mathcal{B}_{r}}(0)=\overline{\mathcal{B}_{r}}$.

Всюду далее $\mathcal{O}$ - непустое открытое множество в $\mathbb{C}^{n}$. Для $r \geqslant 0$ через $\mathcal{O}_{r}$ обозначено множество центров всех замкнутых шаров радиусом $r$, содержащихся в $\mathcal{O}$.

Множество $\mathcal{O}$ называется $\delta$-областъю, если выполнены следующие условия:

(i) каждая точка из $\mathcal{O}$ лежит в некотором замкнутом содержащемся в $\mathcal{O}$ шаре радиусом $\delta$;

(ii) множество $\mathcal{O}_{\delta}$ является связным.

Из определения видно, что при $\delta=0$ понятия $\delta$-области и обычной области в $\mathbb{C}^{n}$ совпадают.

Пусть $\mathcal{D}^{\prime}(\mathcal{O}), \mathcal{E}^{\prime}(\mathcal{O})$ - соответственно пространства распределений и распределений с компактными носителями в $\mathcal{O}, \mathcal{D}(\mathcal{O})$ - пространство финитных бесконечно дифференцируемых функций в $\mathcal{O}$. В случае, когда $\mathcal{O}$ инвариантно относительно унитарной группы $\mathrm{U}(n)$, через $\mathcal{D}_{\natural}^{\prime}(\mathcal{O}), \mathcal{E}_{\natural}^{\prime}(\mathcal{O})$ и $\mathcal{D}_{\natural}(\mathcal{O})$ обозначим соответственно подмножества $\mathcal{D}^{\prime}(\mathcal{O}), \mathcal{E}^{\prime}(\mathcal{O})$ и $\mathcal{D}(\mathcal{O})$, состоящие из всех $\mathrm{U}(n)$-инвариантных распределений. Для $T \in \mathcal{E}^{\prime}\left(\mathbb{C}^{n}\right)$ через $\operatorname{supp} T$ обозначим носитель $T$ и положим

$$
r(T)=\inf \left\{r>0: \operatorname{supp} T \subset \mathcal{B}_{r}\right\}
$$

(C) В. В. Волчков, Вит. В. Волчков, 2012 
Искаженной сверткой (twisted convolution) распределений $f \in \mathcal{D}^{\prime}\left(\mathbb{C}^{n}\right)$ и $T \in$ $\mathcal{E}^{\prime}\left(\mathbb{C}^{n}\right)$ называется распределение $f \star T \in \mathcal{D}^{\prime}\left(\mathbb{C}^{n}\right)$, задаваемое формулой

$$
\langle f \star T, \psi\rangle=\left\langle f(z),\left\langle T(w), \psi(z+w) e^{\frac{i}{2} \operatorname{Im}\langle z, w\rangle_{\mathbb{C}}}\right\rangle\right\rangle, \quad \psi \in \mathcal{D}\left(\mathbb{C}^{n}\right) .
$$

Таким образом, искаженная свертка $f \star T$ отличается от обычной свертки $f * T$ распределений $f$ и $T$ наличием множителя $e^{\frac{i}{2} \operatorname{Im}\langle z, w\rangle_{\mathbb{C}}}$ в правой части равенства (1). Из (1) следует, что если $f \in L^{1, \text { loc }}\left(\mathbb{C}^{n}\right)$ и $T \in\left(\mathcal{E}^{\prime} \cap L^{1, \text { loc }}\right)\left(\mathbb{C}^{n}\right)$, то $f \star T \in L^{1, \text { loc }}\left(\mathbb{C}^{n}\right)$ и

$$
(f \star T)(z)=\int_{\mathbb{C}^{n}} f(z-w) T(w) e^{\frac{i}{2} \operatorname{Im}\langle z, w\rangle_{\mathbb{C}}} d m_{n}(w),
$$

где $d m_{n}(w)$ - мера Лебега на $\mathbb{C}^{n}$.

Пусть $T \in \mathcal{E}_{\natural}^{\prime}\left(\mathbb{C}^{n}\right)$. Предположим, что $\mathcal{O}$ - открытое подмножество в $\mathbb{C}^{n}$, для которого $\mathcal{O}_{r(T)} \neq \varnothing$. Тогда для любого $f \in \mathcal{D}^{\prime}(\mathcal{O})$ свертка корректно определена посредством (1) как распределение в $\mathcal{O}_{r(T)}$. Рассмотрим искаженное уравнение свертки

$$
f \star T=0 \quad \text { в } \quad \mathcal{O}_{r(T)}
$$

с неизвестным $f$. Обозначим через $\mathcal{D}_{T}^{\prime}(\mathcal{O})$ множество всех $f \in \mathcal{D}^{\prime}(\mathcal{O})$, удовлетворяющих (2). Интерес к изучению уравнений вида (2) объясняется в первую очередь тем, что они тесно связаны с группой Гейзенберга, которая широко используется в современных исследованиях по гармоническому анализу и уравнениях в частных производных (см., например, [1]-[3]).

В настоящей работе исследуется

ПробЛема 1. Пусть $T \in \mathcal{E}_{\natural}^{\prime}\left(\mathbb{C}^{n}\right), T \neq 0$, и пусть $\mathcal{O}$ - неограниченная $\delta$-область в $\mathbb{C}^{n}$ при $\delta=r(T)$. Предположим, ито $f \in\left(L^{1, \text { lос }} \cap \mathcal{D}_{T}^{\prime}\right)(\mathcal{O})$. При каких ограничениях, налагаемых на рост распределения $f$ в бесконечности, отсюда следует, что $f=0$ ?

Отметим, что условие, налагаемое на $\mathcal{O}$ в проблеме 1 , является естественным, поскольку для произвольных областей $\mathcal{O}$, для которых $\mathcal{O}_{r(T)} \neq \varnothing$, уравнение $(2)$ может не содержать каких-либо ограничений для $f$ в некотором открытом подмножестве из $\mathcal{O}$.

Аналоги проблемы 1 для уравнений с обычной сверткой на областях в вещественном евклидовом пространстве $\mathbb{R}^{n}$ изучаются более семидесяти лет начиная с классического результата Ф. Йона о сферических средних (см. [4, гл. 6]). Однако, несмотря на активные исследования в этой области, полученных точных результатов немного. Все они касаются случаев, когда $\mathcal{O}=\mathbb{R}^{n}, \mathcal{O}$ - внешность компакта или $\mathcal{O}$ - некоторый класс областей, содержащих полупространство (см. [5]-[14]).

В отличие от случая уравнений с обычной сверткой, проблема 1 представляется гораздо более трудной. В значительной мере это объясняется существенным нарушением групповой структуры сдвигов, играющей важную роль при изучении оператора свертки, и, соответственно, невозможностью применения методов, связанных с преобразованием Фурье. В частности, до настоящего времени не было известно ни одного нетривиального результата по проблеме 1 для 
областей $\mathcal{O}$, отличных от $\mathbb{C}^{n}$. В случае $\mathcal{O}=\mathbb{C}^{n}$ наиболее сильный (однако, как теперь выяснилось, неточный) результат принадлежит С. Сангавелу [15]: если $T \in \mathcal{E}_{\natural}^{\prime}\left(\mathbb{C}^{n}\right)$ является мерой и сферическое преобразование $\widetilde{T}$ не имеет нулей $\lambda$, удовлетворяющих условию

$$
\frac{n-\lambda^{2}}{2} \in\{0,-1,-2, \ldots\},
$$

то всякое непрерывное решение уравнения (2) на $\mathbb{C}^{n}$ умеренного роста равно нулю тождественно. Здесь и далее сферическое преобразование $\widetilde{T}$ распределения $T \in \mathcal{E}_{\natural}^{\prime}\left(\mathbb{C}^{n}\right)$ определяется равенством

$$
\widetilde{T}(\lambda)=\left\langle T(z), e^{-\frac{|z|^{2}}{4}}{ }_{1} F_{1}\left(\frac{n-\lambda^{2}}{2} ; n ; \frac{|z|^{2}}{2}\right)\right\rangle,
$$

где ${ }_{1} F_{1}$ - вырожденная гипергеометрическая функция (см. [16, гл. 6]). Функция $\widetilde{T}$ является целой и четной. Условие (3) в теореме С. Сангавелу убрать нельзя. Доказательство теоремы С. Сангавелу основано на применении преобразования Вейля, которое является аналогом преобразования Фурье в $\mathbb{R}^{n}$ на пространстве распределений умеренного роста (см. [1], [2], [15]).

В последние годы с помощью предложенной авторами новой техники, основанной на трансмутационных отображениях, был достигнут заметный прогресс в изучении уравнений типа (2) (см. [17]). Применение этой техники в настоящей работе позволило добиться продвижения и в проблеме 1.

Основные результаты работы заключаются в следующем.

1. Для случая $\mathcal{O}=\mathbb{C}^{n}$ получено существенное уточнение и обобщение сформулированной выше теоремы С. Сангавелу. В частности, условие умеренного роста для решений уравнения (2) из этой теоремы ослаблено до экспоненциального роста с квадратичным полиномом в показателе экспоненты (см. теоремy 1).

2. Впервые изучается случай, когда $\mathcal{O}$ является внешностью компакта в $\mathbb{C}^{n}$ (см. теорему 2).

3. Показано, что упомянутые выше теоремы 1, 2 являются, в определенном смысле, окончательными (см. теорему 3).

Некоторые промежуточные результаты работы представляют самостоятельный интерес (см. леммы 4, 5 в §5). В частности, лемма 4, возможно, окажется полезной при изучении проблемы 1 для иных областей $\mathcal{O}$.

Результаты, касающиеся проблемы 1 и ее аналогов для других классов функций, можно найти в [8], [18], [19], [20, гл. 3, § 3.4, 3.5] (см. также библиографию к этим работам).

\section{§ 2. Формулировки основных результатов}

Положим

$$
E(R, r)=\left\{z \in \mathbb{C}^{n}: R-r<|z|<R+r\right\}, \quad 0<r<R .
$$

Для распределения $T \in \mathcal{E}_{\natural}^{\prime}\left(\mathbb{C}^{n}\right)$ обозначим через $N_{T}$ множество нулей $\lambda$ 
функции $\widetilde{T}$, удовлетворяющих условию (3). Пусть

$$
\operatorname{RA}_{T}(\mathcal{O})=\left(\mathcal{D}_{T}^{\prime} \cap \mathrm{RA}\right)(\mathcal{O})
$$

где $\mathrm{RA}(\mathcal{O})$ - множество всех вещественно-аналитических функций на $\mathcal{O}$.

Tеорема 1. Пустъ $T \in \mathcal{E}_{\natural}^{\prime}\left(\mathbb{C}^{n}\right), T \neq 0$, u nycmъ $f \in\left(L^{1, \text { loc }} \cap \mathcal{D}_{T}^{\prime}\right)\left(\mathbb{C}^{n}\right)$. Тогда имеют место следующие утверждения:

(i) если $N_{T}=\varnothing$ и для некоторых $\gamma>r(T), \eta<\frac{1}{2}(\gamma-r(T))$

$$
\lim _{R \rightarrow+\infty} e^{-\frac{R^{2}}{4}-\eta R} \int_{E(R, \gamma)}|f(z)| d m_{n}(z)=0,
$$

mo $f=0$;

(ii) если $N_{T} \neq \varnothing$ и для некоторых $\gamma>r(T), \eta<\frac{1}{2}(\gamma-r(T))$

$$
\varliminf_{R \rightarrow+\infty} e^{\frac{R^{2}}{4}-\eta R} \int_{E(R, \gamma)}|f(z)| d m_{n}(z)=0,
$$

$m o f=0$.

Рассмотрим теперь случай, когда $\mathcal{O}$ - внешность компакта.

Tеорема 2. Пусть $T \in \mathcal{E}_{\natural}^{\prime}\left(\mathbb{C}^{n}\right), T \neq 0$, и nустъ $\mathcal{O}-\delta$-область в $\mathbb{C}^{n}$, где $\delta=r(T)$, которая является внешностью некоторого компакта в $\mathbb{C}^{n}$. Предположим, что $f \in\left(L^{1, \text { loc }} \cap \mathcal{D}_{T}^{\prime}\right)(\mathcal{O})$ и для некоторых $\gamma>r(T), \eta<\frac{1}{2}(\gamma-r(T))$ выполнено условие (6). Тогда $f=0$.

Отметим, что утверждение (ii) теоремы 1 следует из теоремы 2, если положить в последней $\mathcal{O}=\mathbb{C}^{n} \backslash\{0\}$.

Следующий результат показывает, что теоремы 1, 2 носят окончательный характер.

ТЕОРема 3. Пусть $T \in \mathcal{E}_{\natural}^{\prime}\left(\mathbb{C}^{n}\right)$. Тогда выполнены следующие утверждения:

(i) для любых $\gamma>0, \eta>\frac{\gamma}{2}$ существует ненулевая функиия $f \in \mathrm{RA}_{T}\left(\mathbb{C}^{n}\right)$, для которой

$$
\int_{E(R, \gamma)}|f(z)| d m_{n}(z)=O\left(e^{\frac{R^{2}}{4}+\eta R}\right), \quad R \rightarrow+\infty
$$

(ii) если $N_{T} \neq \varnothing$, то для любых $\gamma>0, \eta>\frac{\gamma}{2}$ существует ненулевая функиия $f \in \mathrm{RA}_{T}\left(\mathbb{C}^{n}\right)$ такая, что

$$
\int_{E(R, \gamma)}|f(z)| d m_{n}(z)=O\left(e^{-\frac{R^{2}}{4}+\eta R}\right), \quad R \rightarrow+\infty
$$

(iii) для любых $\gamma>0, \eta>\frac{\gamma}{2}$ существует ненулевая функиия $f \in \mathrm{RA}_{T}\left(\mathbb{C}^{n} \backslash\right.$ $\{0\})$, удовлетворяющая условию (8).

Сравнивая теоремы 1, 2, 3, получим, что главные члены в показателях экспонент в условиях (5) и (6) являются точными. 


\section{§ 3. Обозначения и вспомогательные конструкции}

Далее используются следующие стандартные обозначения: $\mathbb{C}$ - множество комплексных чисел; $\mathbb{Z}_{+}=\{0,1,2, \ldots\} ;\left(\begin{array}{l}j \\ k\end{array}\right)$ - биномиальные коэффициенты; $\Gamma$ гамма-функция; $\mathcal{D}(a, b)$ - множество финитных бесконечно дифференцируемых функций на интервале $(a, b)$. Если $T$ - распределение с компактным носителем в $\mathbb{R}$, то через $\widehat{T}$ обозначается его преобразование Фурье, т. е.

$$
\widehat{T}(z)=\left\langle T(t), e^{-i t z}\right\rangle, \quad z \in \mathbb{C} .
$$

Пусть

$$
\mathcal{D}_{\natural}(-R, R)=\{f \in \mathcal{D}(-R, R): f(-t)=f(t)\}, \quad \mathcal{Z}(f)=\{z \in \mathbb{C}: f(z)=0\}
$$

для функции $f: \mathbb{C} \rightarrow \mathbb{C}$.

Пусть $\mathcal{H}^{n, p, q}$ - пространство сферических гармоник бистепени $(p, q)$ на $\mathbb{S}^{2 n-1}$ (см. [21, гл. 12]). Как известно [21, теоремы 12.2.7, 12.2.8], квазирегулярное представление группы $\mathrm{U}(n)$ в $L^{2}\left(\mathbb{S}^{2 n-1}\right)$ является прямой суммой попарно неэквивалентных неприводимых унитарных представлений, действующих на $\mathcal{H}^{n, p, q}$. Обозначим через $d(n, p, q)$ размерность $\mathcal{H}^{n, p, q}$, и пусть $\left\{S_{l}^{p, q}\right\}$, $l \in\{1, \ldots, d(n, p, q)\},-$ фиксированный ортонормированный базис в $\mathcal{H}^{n, p, q}$. Положим $S_{1}^{0,0}(\sigma)=\frac{1}{\sqrt{\omega_{2 n-1}}}$ для любого $\sigma \in \mathbb{S}^{2 n-1}$, где $\omega_{2 n-1}=\frac{2 n \pi^{n}}{\Gamma(n+1)}-$ площадь сферы $\mathbb{S}^{2 n-1}$. Всякой функции $f \in L^{1, \text { lос }}(\mathcal{O})$, где $\mathcal{O}$ - непустое открытое $\mathrm{U}(n)$-инвариантное множество в $\mathbb{C}^{n}$, соответствует ряд Фурье

$$
f(z) \sim \sum_{p, q=0}^{\infty} \sum_{l=1}^{d(n, p, q)} f_{p, q, l}(\varrho) S_{l}^{p, q}(\sigma), \quad z=\varrho \sigma, \quad \varrho=|z|, \quad \sigma \in \mathbb{S}^{2 n-1},
$$

где

$$
f_{p, q, l}(\varrho)=\int_{\mathbb{S}^{2 n-1}} f(\varrho \sigma) \overline{S_{l}^{p, q}(\sigma)} d \omega(\sigma) .
$$

Разложение (9) можно продолжить на распределения $f \in \mathcal{D}^{\prime}(\mathcal{O})$ следующим образом:

$$
f \sim \sum_{p, q=0}^{\infty} \sum_{l=1}^{d(n, p, q)} f^{p, q, l}
$$

где распределение $f^{p, q, l}$ действует по правилу

$$
\left\langle f^{p, q, l}, \psi\right\rangle=\left\langle f, \overline{(\bar{\psi})_{p, q, l}(\varrho)} \overline{S_{l}^{p, q}(\sigma)}\right\rangle, \quad \psi \in \mathcal{D}(\mathcal{O}) .
$$

Для заданного класса $\mathfrak{W}(\mathcal{O})$ распределений на $\mathcal{O}$ положим

$$
\mathfrak{W}_{p, q, l}(\mathcal{O})=\left\{f \in \mathfrak{W}(\mathcal{O}): f=f^{p, q, l}\right\} .
$$

Если $T \in \mathcal{E}_{\natural}^{\prime}\left(\mathbb{C}^{n}\right), r(T)<R \leqslant+\infty$ и $f \in \mathcal{D}^{\prime}\left(\mathcal{B}_{R}\right)$, то из (1) и (11) следует, что

$$
(f \star T)^{p, q, l}=f^{p, q, l} \star T \quad \text { в } \quad \mathcal{B}_{R-r(T)} .
$$


Кроме того,

$$
f \star \delta_{0}=\delta_{0} \star f=f \quad \text { в } \quad \mathcal{B}_{R},
$$

где $\delta_{0}-$ мера Дирака в нуле.

Введем специальный оператор Эрмита (искаженный лапласиан)

$$
\mathfrak{L}=\frac{|z|^{2}}{4} \operatorname{Id}+\sum_{k=1}^{n}\left(z_{k} \frac{\partial}{\partial z_{k}}-\bar{z}_{k} \frac{\partial}{\partial \bar{z}_{k}}-4 \frac{\partial^{2}}{\partial z_{k} \partial \bar{z}_{k}}\right)
$$

где Id - тождественный оператор. Если $f \in C_{p, q, l}^{2}(\mathcal{O})$, то из $(13)$ непосредственным вычислением получается соотношение

$$
(\mathfrak{L} f)(z)=\left(\mathfrak{L}_{p, q} f_{p, q, l}\right)(\varrho) S_{l}^{p, q}(\sigma),
$$

где

$$
\mathfrak{L}_{p, q}=-\frac{d^{2}}{d \varrho^{2}}-\frac{2 n-1}{\varrho} \frac{d}{d \varrho}+\left(\frac{(p+q)(2 n+p+q-2)}{\varrho^{2}}+\frac{1}{4} \varrho^{2}+p-q\right) \text { Id } .
$$

Далее, пусть $T_{j} \in \mathcal{D}^{\prime}\left(\mathbb{C}^{n}\right), j=1,2,3$, и хотя бы два из распределений $T_{j}$ имеют компактный носитель. Тогда из определения искаженной свертки следуют равенства

$$
\begin{aligned}
&\left(\lambda T_{1}+\mu T_{2}\right) \star T_{3}=\lambda\left(T_{1} \star T_{3}\right)+\mu\left(T_{2} \star T_{3}\right), \quad \lambda, \mu \in \mathbb{C}, \\
&\left(T_{1} \star T_{2}\right) \star T_{3}=T_{1} \star\left(T_{2} \star T_{3}\right)
\end{aligned}
$$

и имеет место вложение

$$
\operatorname{supp}\left(T_{1} \star T_{2}\right) \subset \operatorname{supp} T_{1}+\operatorname{supp} T_{2} .
$$

Кроме того, используя легко проверяемое тождество

$$
\mathfrak{L}\left(h(z+w) e^{\frac{i}{2} \operatorname{Im}\langle z, w\rangle_{\mathbb{C}}}\right)=(\mathfrak{L} h)(z+w) e^{\frac{i}{2} \operatorname{Im}\langle z, w\rangle_{\mathbb{C}}}, \quad h \in C^{2}\left(\mathbb{C}^{n}\right),
$$

получаем, что

$$
\mathfrak{L}\left(T_{1} \star T_{2}\right)=T_{1} \star \mathfrak{L} T_{2}
$$

\section{§ 4. Функции $\phi_{\lambda, p, q, l}$ и $\psi_{\lambda, p, q, l}$}

Пусть $\Phi(a, b ; \zeta)$ и $\Psi(a, b ; \zeta)$ - вырожденные гипергеометрические функции Куммера и Трикоми соответственно [16, гл. 6]. Эти функции удовлетворяют уравнению

$$
\zeta u^{\prime \prime}(\zeta)+(b-\zeta) u^{\prime}(\zeta)-a u(\zeta)=0 .
$$

Пусть $\lambda \in \mathbb{C}, p, q \in \mathbb{Z}_{+}, l \in\{1, \ldots, d(n, p, q)\}$. Положим

$$
a=p+\frac{n-\lambda^{2}}{2}, \quad b=n+p+q .
$$

Для $z=\varrho \sigma \in \mathbb{C}^{n} \backslash\{0\}$ определим

$$
\phi_{\lambda, p, q, l}(z)=\sqrt{\omega_{2 n-1}} \phi_{\lambda, p, q}(\varrho) S_{l}^{p, q}(\sigma),
$$


где

$$
\phi_{\lambda, p, q}(\varrho)=\varrho^{p+q} e^{-\frac{\varrho^{2}}{4}} \Phi\left(a, b ; \frac{\varrho^{2}}{2}\right) .
$$

Функция $\phi_{\lambda, p, q, l}$ допускает непрерывное продолжение в точку $z=0$. Доопределяя $\phi_{\lambda, p, q, l}$ в нуле по непрерывности, получаем, что $\phi_{\lambda, p, q, l} \in \mathrm{RA}\left(\mathbb{C}^{n}\right)$.

Далее, при $\varrho \in(0,+\infty)$ положим

$$
\psi_{\lambda, p, q}(\varrho)= \begin{cases}\varrho^{p+q} e^{-\frac{\varrho^{2}}{4}} \Psi\left(a, b ; \frac{\varrho^{2}}{2}\right), & \text { если }-a \notin \mathbb{Z}_{+}, \\ \varrho^{p+q} e^{\frac{\varrho^{2}}{4}} \Psi\left(b-a, b ;-\frac{\varrho^{2}}{2}\right), & \text { если }-a \in \mathbb{Z}_{+} .\end{cases}
$$

Теперь для $z=\varrho \sigma \in \mathbb{C}^{n} \backslash\{0\}$ определим

$$
\psi_{\lambda, p, q, l}(z)=\psi_{\lambda, p, q}(\varrho) S_{l}^{p, q}(\sigma) .
$$

Таким образом, $\psi_{\lambda, p, q, l} \in \mathrm{RA}\left(\mathbb{C}^{n} \backslash\{0\}\right)$.

Лемма 1. Пусть $w \in \mathcal{D}_{p, q, l}^{\prime}\left(\mathbb{C}^{n} \backslash\{0\}\right), \lambda \in \mathbb{C}$. Тогда следующие утверждения эквивалентны:

(i) $\left(\mathfrak{L}-\lambda^{2} \mathrm{Id}\right) w=0$;

(ii) $w=c_{1} \phi_{\lambda, p, q, l}+c_{2} \psi_{\lambda, p, q, l}$.

ДокАЗАтЕльство. В силу эллиптичности оператора $\mathfrak{L}$ достаточно доказать лемму для вещественно-аналитических $w$. Полагая $w(z)=f(\varrho) S_{l}^{p, q}(\sigma)$ и используя (14), перепишем уравнение $\left(\mathfrak{L}-\lambda^{2} \mathrm{Id}\right) w=0$ в виде

$f^{\prime \prime}(\varrho)+\frac{f^{\prime}(\varrho)}{\varrho}(2 n-1)-\frac{f(\varrho)}{\varrho^{2}}\left((p+q)(2 n+p+q-2)+\left(p-q-\lambda^{2}\right) \varrho^{2}+\frac{1}{4} \varrho^{4}\right)=0$.

Делая замену

$$
f(\varrho)=\varrho^{p+q} e^{-\frac{\varrho^{2}}{4}} u\left(\frac{\varrho^{2}}{2}\right)
$$

приходим к вырожденному гипергеометрическому уравнению (19).

Если $-a \notin \mathbb{Z}_{+}$, функции $\Phi(a, b ; \zeta)$ и $\Psi(a, b ; \zeta)$ образуют фундаментальную систему решений уравнения (19) (см. [16, гл. 6, §6.7]). При $-a \in \mathbb{Z}_{+}$эти функции отличаются ненулевым постоянным множителем и вторым линейно независимым решением (19) является функция $e^{\zeta} \Psi(b-a, b ; \zeta)$ (см. [16, гл. 6, $\S 6.7])$. Учитывая определения для $\phi_{\lambda, p, q, l}$ и $\psi_{\lambda, p, q, l}$, отсюда получаем утверждение леммы.

СлЕДСтвиЕ 1. Множество всех $w \in \mathcal{D}_{p, q, l}^{\prime}\left(\mathbb{C}^{n}\right)$, удовлетворяющих уравнению

$$
\left(\mathfrak{L}-\lambda^{2} \operatorname{Id}\right) w=0,
$$

uмеет вид $w=c \phi_{\lambda, p, q, l}$, где $c \in \mathbb{C}$.

ДокАЗАТЕЛЬство следует из леммы 1 и того, что функция $\psi_{\lambda, p, q, l}$ имеет особенность в точке $z=0$ (см. [16, гл. $6, \S 6.8]$ ). 
Рассмотрим теперь асимптотическое поведение функций $\phi_{\lambda, p, q}$ и $\psi_{\lambda, p, q}$ при $\varrho \rightarrow+\infty$.

ЛЕмма 2. Имеют место следующие утверждения:

(i) если $-a \notin \mathbb{Z}_{+}$, mo

$$
\begin{gathered}
\phi_{\lambda, p, q}(\varrho)=2^{b-a} \frac{\Gamma(b)}{\Gamma(a)} \varrho^{p+q+2(a-b)} e^{\frac{\varrho^{2}}{4}}\left(1+O\left(\frac{1}{\varrho}\right)\right), \quad \varrho \rightarrow+\infty \\
\psi_{\lambda, p, q}(\varrho)=2^{a} \varrho^{p+q-2 a} e^{-\frac{\varrho^{2}}{4}}\left(1+O\left(\frac{1}{\varrho}\right)\right), \quad \varrho \rightarrow+\infty
\end{gathered}
$$

(ii) если $-a \in \mathbb{Z}_{+}$, mo

$$
\begin{gathered}
\phi_{\lambda, p, q}(\varrho)=(-2)^{a} \frac{\Gamma(b)}{\Gamma(b-a)} \varrho^{p+q-2 a} e^{-\frac{\varrho^{2}}{4}}\left(1+O\left(\frac{1}{\varrho}\right)\right), \quad \varrho \rightarrow+\infty \\
\psi_{\lambda, p, q}(\varrho)=(-2)^{b-a} \varrho^{p+q+2(a-b)} e^{\frac{\varrho^{2}}{4}}\left(1+O\left(\frac{1}{\varrho}\right)\right), \quad \varrho \rightarrow+\infty
\end{gathered}
$$

ДокАзАтельство. Утверждение (i) следует из определения функций $\phi_{\lambda, p, q}$ и $\psi_{\lambda, p, q}$ и асимптотических формул для $\Phi(a, b ; \zeta)$ и $\Psi(a, b ; \zeta)$ при $\zeta \rightarrow+\infty$ (см. $[16$, гл. $6, \S 6.13])$. В случае, когда $-a \in \mathbb{Z}_{+}$, функция $\Phi(a, b ; \zeta)$ является многочленом степени $-a$ со старшим коэффициентом, равным

$$
(-1)^{-a} \frac{\Gamma(b)}{\Gamma(b-a)}
$$

(см. [16, гл. 6, § 6.1, формула (1)]). Отсюда и из асимптотической формулы для $\Psi(b-a, b ; \zeta)$ при $\zeta \rightarrow+\infty$ (см. [16, гл. $6, \S 6.13])$ получаем утверждение (ii).

Далее, для любого $f \in \mathcal{E}_{p, q, l}^{\prime}\left(\mathbb{C}^{n}\right)$ положим

$$
\mathcal{F}_{l}^{p, q}(f)(\lambda)=\left\langle f, \overline{\phi_{\bar{\lambda}, p, q, l}}\right\rangle=\sqrt{\omega_{2 n-1}}\left\langle f, \phi_{\lambda, p, q}(\varrho) \overline{S_{l}^{p, q}(\sigma)}\right\rangle, \quad \lambda \in \mathbb{C} .
$$

Согласно $(24)$ функция $\mathcal{F}_{l}^{p, q}(f)$ является четной целой функцией переменной $\lambda$. Если $f \in \mathcal{E}_{\natural}^{\prime}\left(\mathbb{C}^{n}\right)$, функция $\mathcal{F}_{1}^{0,0}(f)(\lambda)$ совпадает со сферическим преобразованием $f$, т. е.

$$
\mathcal{F}_{1}^{0,0}(f)(\lambda)=\tilde{f}(\lambda)
$$

Преобразование $\mathcal{F}_{l}^{p, q}$ хорошо изучено в работе $[17, \S 6]$. Напомним некоторые основные свойства $\mathcal{F}_{l}^{p, q}$, которые потребуются в дальнейшем.

Лемма 3. (i) Преобразование $\mathcal{F}_{l}^{p, q}$ является инбективным на $\mathcal{E}_{p, q, l}^{\prime}\left(\mathbb{C}^{n}\right)$.

(ii) Ecлu $f \in \mathcal{E}_{p, q, l}^{\prime}\left(\mathbb{C}^{n}\right) u T \in \mathcal{E}_{\natural}^{\prime}\left(\mathbb{C}^{n}\right)$, mo

$$
\mathcal{F}_{l}^{p, q}(f \star T)(\lambda)=\mathcal{F}_{l}^{p, q}(f)(\lambda) \widetilde{T}(\lambda) .
$$

(iii) Пусть $f \in \mathcal{E}_{p, q, l}^{\prime}\left(\mathbb{C}^{n}\right) u \operatorname{supp} f \subset \overline{\mathcal{B}_{r}}$. Тогда

$$
\left|\mathcal{F}_{l}^{p, q}(f)(\lambda)\right| \leqslant c_{1}(1+|\lambda|)^{c_{2}} e^{r|\operatorname{Im} \lambda|} \quad \forall \lambda \in \mathbb{C},
$$


где $c_{1}, c_{2}>0$ не зависят от $\lambda$. Обратно, для каждой четной иелой функции $w(\lambda)$, удовлетворяющей ощенке вида (26), существует такое распределенuе $f \in \mathcal{E}_{p, q, l}^{\prime}\left(\mathbb{C}^{n}\right)$, чmo

$$
\operatorname{supp} f \subset \overline{\mathcal{B}_{r}}, \quad \mathcal{F}_{l}^{p, q}(f)=w \text {. }
$$

ДокАЗАТЕЛЬСТво леммы и более подробную информацию о свойствах преобразования $\mathcal{F}_{l}^{p, q}$ см. в $[17, \S 6]$.

\section{§5. Некоторые свойства решений искаженного уравнения свертки}

При доказательстве основных результатов работы важную роль играют следующие вспомогательные утверждения, представляющие самостоятельный интерес.

Лемма 4. Пусть $T \in \mathcal{E}_{\natural}^{\prime}\left(\mathbb{C}^{n}\right), T \neq 0$, и пусть $\mathcal{O}$ - произвольная область в $\mathbb{C}^{n}$, для которой $\mathcal{O}_{r(T)} \neq 0$. Предположим, ито $f \in\left(L^{1, \text { loc }} \cap \mathcal{D}_{T}^{\prime}\right)(\mathcal{O})$ u $f \neq 0$. Тогда существует ненулевая функиия $g \in C^{\infty}\left(\mathcal{O}_{r(T)}\right)$, удовлетворяющая следующим условиям:

1) $\mathfrak{L} g=\lambda^{2} g$ в $\mathcal{O}_{r(T)}$ для некоторого $\lambda \in \mathcal{Z}(\widetilde{T})$;

2) для любого $\varepsilon>0$ и любого замкнутого шара $\overline{\mathcal{B}}_{r(T)+\varepsilon}(z) \subset \mathcal{O}$

$$
|g(z)| \leqslant c \int_{\overline{\mathcal{B}}_{r(T)+\varepsilon}(z)}|f(w)| d m_{n}(w),
$$

где постоянная $c>0$ не зависит от $z$.

Доказательство. Сначала докажем, что $\mathcal{Z}(\widetilde{T}) \neq \varnothing$. Действительно, в противном случае $\widetilde{T}$ является четной целой функцией экспоненциального типа, не имеющей нулей (см. лемму 3, (iii)). Тогда в силу теоремы Адамара о факторизации целых функций распределение $\widetilde{T}$ является тождественной константой. Это означает, что $T=c_{1} \delta_{0}$ для некоторого $c_{1} \in \mathbb{C}, c_{1} \neq 0$. Поэтому $\mathcal{O}_{T}=\mathcal{O}$ и $f \star T=c_{1} f$ в $\mathcal{O}$, что противоречит условиям $f \in \mathcal{D}_{T}^{\prime}(\mathcal{O})$ и $f \neq 0$. Таким образом, $\mathcal{Z}(\widetilde{T}) \neq \varnothing$.

Далее, используя лемму 3 , (iii), для любого $\lambda \in \mathcal{Z}(\widetilde{T})$ определим распределение $T_{\lambda} \in \mathcal{E}_{\natural}^{\prime}\left(\mathbb{C}^{n}\right)$ по формуле

$$
\widetilde{T}_{\lambda}(z)= \begin{cases}\frac{\widetilde{T}(z)}{\left(z^{2}-\lambda^{2}\right)^{m(\lambda, T)}}, & \text { если } \lambda \neq 0, \\ \frac{\widetilde{T}(z)}{z^{m(0, T)}}, & \text { если } \lambda=0 \in \mathcal{Z}(\widetilde{T}),\end{cases}
$$

где $m(\lambda, T)$ - кратность нуля $\lambda$ функции $\widetilde{T}$. Из (29) и леммы 3 , (iii) следует, что $r\left(T_{\lambda}\right)=r(T)$.

Докажем теперь, что $f \star T_{\lambda} \neq 0$ в $\mathcal{O}_{T}$ для некоторого $\lambda \in \mathcal{Z}(\widetilde{T})$. Не ограничивая общности, можно считать, что $\mathcal{O}=\mathcal{B}_{R}, R>r(T)$. Предположим противное, т. е.

$$
f \star T_{\lambda}=0 \quad \text { в } \quad \mathcal{B}_{R-r(T)} \quad \forall \lambda \in \mathcal{Z}(\widetilde{T}) .
$$


Сначала рассмотрим случай, когда $f \in \mathcal{D}_{p, q, l}^{\prime}\left(\mathcal{B}_{R}\right)$ при некоторых $p, q, l$. Для $\psi \in \mathcal{D}(-R, R)$ выберем $\eta \in \mathcal{D}_{\natural}\left(\mathcal{B}_{R}\right)$ такое, что $\eta=1$ в $\mathcal{B}_{r(\psi)+\delta}$ при некотором $\delta \in$ $(0, R-r(\psi))$, где

$$
r(\psi)=\inf \{r>0: \operatorname{supp} \psi \subset(-r, r)\}
$$

Положим

$$
\left\langle\mathfrak{A}_{p, q, l}(f), \psi\right\rangle=\sum_{j=0}^{\infty} \mu_{j} \mathcal{F}_{l}^{p, q}(f \eta)\left(\lambda_{j}\right) \int_{-R}^{R} \psi(t) \cos \left(\lambda_{j} t\right) d t
$$

где

$$
\lambda_{j}=\sqrt{2 p+n+2 j}, \quad \mu_{j}=\frac{2^{1-n-p-q}}{\omega_{2 n-1}(n+p+q-1) !}\left(\begin{array}{c}
n+p+q+j-1 \\
n+p+q-1
\end{array}\right) .
$$

Тогда $\mathfrak{A}_{p, q, l}(f)$ корректно определено посредством $(31)$ как распределение в $\mathcal{D}_{\natural}^{\prime}(-R, R)$ и

$$
\mathfrak{A}_{p, q, l}\left(\left.f\right|_{\mathcal{B}_{r}}\right)=\left.\mathfrak{A}_{p, q, l}(f)\right|_{(-r, r)}
$$

для любого $r \in(0, R]$ (см. [17, § 7]). Кроме того, из $(31)$ и [17, теорема 9] имеем

$$
\mathfrak{A}_{p, q, l}\left(f \star T_{\lambda}\right)=\mathfrak{A}_{p, q, l}(f) * \Lambda\left(T_{\lambda}\right)=0
$$

на $(r(T)-R, R-r(T))$, где распределение $\Lambda\left(T_{\lambda}\right) \in \mathcal{E}_{\natural}^{\prime}(\mathbb{R})$ определяется равенством

$$
\widehat{\Lambda\left(T_{\lambda}\right)}(z)=\widetilde{T_{\lambda}}(z), \quad z \in \mathbb{C} .
$$

Далее, используя [17, теорема 4], из (33) заключаем, что $\mathfrak{A}_{p, q, l}(f)=0$. Тогда в силу инъективности преобразования $\mathfrak{A}_{p, q, l}$ (см. $[17$, теорема 9]) имеем $f=0$, что противоречит условию леммы. Рассмотрим теперь общий случай. Из соотношений (30) и (12) получаем, что

$$
f^{p, q, l} \star T_{\lambda}=0 \quad \text { в } \quad \mathcal{B}_{R-r(T)} \quad \forall \lambda, p, q, l .
$$

По уже доказанному имеем $f^{p, q, l}=0$. Отсюда в силу произвольности выбоpa $p, q, l$ имеем $f=0$, что также противоречит условию. Итак, существует $\lambda \in \mathcal{Z}(\widetilde{T})$ такое, что $f \star T_{\lambda} \neq 0$ в $\mathcal{O}_{T}$. Для такого $\lambda$ из (29) получаем

$$
T=\left\{\begin{array}{lll}
\left(\mathfrak{L}-\lambda^{2} \mathrm{Id}\right)^{m(\lambda, T)} T_{\lambda}, & \text { если } & \lambda \neq 0, \\
\mathfrak{L}^{m(0, T) / 2} T_{0}, & \text { если } & \lambda=0 .
\end{array}\right.
$$

Тогда для некоторого $\nu \in \mathbb{Z}_{+}$функция $g=\left(\mathfrak{L}-\lambda^{2} \mathrm{Id}\right)^{\nu}\left(f \star T_{\lambda}\right)$ не равна нулю в $\mathcal{O}_{T}$ и удовлетворяет условию 1) леммы.

Пусть теперь $\varepsilon>0$ и $\overline{\mathcal{B}}_{r(T)+\varepsilon}(z) \subset \mathcal{O}$. Тогда для любой функции $\varphi \in \mathcal{D}_{\natural}\left(\mathbb{C}^{n}\right)$ такой, что $\widetilde{\varphi}(\lambda)=1$ и $\operatorname{supp} \varphi \subset \mathcal{B}_{\varepsilon}$, находим

$$
g(z)=(g \star \varphi)(z)=\left(\left(\left(\mathfrak{L}-\lambda^{2} \operatorname{Id}\right)^{\nu}\left(f \star T_{\lambda}\right)\right) \star \varphi\right)(z)=\left(f \star\left(\left(\mathfrak{L}-\lambda^{2} \operatorname{Id}\right)^{\nu} T_{\lambda} \star \varphi\right)\right)(z)
$$


(см. (15), (16), (18)). Учитывая, что $\left(\mathfrak{L}-\lambda^{2} \mathrm{Id}\right)^{\nu} T_{\lambda} \star \varphi \in \mathcal{D}_{\natural}\left(\mathbb{C}^{n}\right)$ и

$$
\operatorname{supp}\left(\left(\mathfrak{L}-\lambda^{2} \mathrm{Id}\right)^{\nu} T_{\lambda} \star \varphi\right) \subset \mathcal{B}_{r(T)+\varepsilon}
$$

(см. (17)), из (37) получаем оценку (28). Тем самым лемма полностью доказана.

Из доказательства леммы 4 видно, что если $T \in \mathcal{D}_{\mathfrak{\natural}}\left(\mathbb{C}^{n}\right)$, то свойство 2) функции $g$ выполнено и при $\varepsilon=0$. Одним из следствий леммы 4 является следующее утверждение.

Лемма 5. Пусть $T \in \mathcal{E}_{\natural}^{\prime}\left(\mathbb{C}^{n}\right), T \neq 0$, и пусть $\mathcal{O}-\delta$-область в $\mathbb{C}^{n}, \delta=r(T)$. Предположим, что $f \in \mathcal{D}_{T}^{\prime}(\mathcal{O})$ u $f=0$ в некотором шаре $\mathcal{B}_{R}(z) \subset \mathcal{O}$, где $R>r(T)$. Тогда $f=0$ в $\mathcal{O}$.

ДокАзАТЕЛЬство. Не ограничивая общности, можно считать, что $f \in$ $C_{T}^{\infty}(\mathcal{O})$. Общий случай будет следовать отсюда в помощью стандартного метода сглаживания (см., например, [14, ч. 1, гл. $3, \S 3.3])$. Предположим, что $f \neq 0$ в $\mathcal{O}$. Пусть $g$ - ненулевая функция, удовлетворяющая условиям 1$)$ и 2 ) из леммы 4. Поскольку $f=0$ в $\mathcal{B}_{R}(z)$, из $(28)$ получаем, что $g=0$ в $\mathcal{B}_{R-r(T)}(z)$. Кроме того, в силу эллиптичности оператора $\mathfrak{L}$ из свойства 1$)$ функции $g$ следует, что $g$ вещественно-аналитична в $\mathcal{O}_{T}$. Таким образом, $g=0$ в $\mathcal{O}_{T}$. Полученное противоречие показывает, что $f=0$ в $\mathcal{O}$.

\section{§ 6. Доказательство основных результатов}

ДокАЗАТЕЛЬСтво теоремЫ 1. Достаточно рассмотреть случай $\eta>0$. Пусть $r(T)<\alpha<\gamma, \beta=\gamma-\alpha$ и $\chi_{\alpha}-$ индикатор шара $\mathcal{B}_{\alpha}$. При $R \geqslant \gamma$ имеем

$$
\begin{aligned}
\int_{E(R, \beta)} \int_{\mathcal{B}_{\alpha}(z)}|f(w)| d m_{n}(w) d m_{n}(z) & \\
\leqslant & \int_{E(R, \beta)} \int_{E(R, \gamma)}|f(w)| \chi_{\alpha}(z-w) d m_{n}(w) d m_{n}(z) \\
\leqslant & \int_{E(R, \gamma)}|f(w)| d m_{n}(w) \int_{\mathbb{C}^{n}} \chi_{\alpha}(z) d m_{n}(z)
\end{aligned}
$$

Предположим, что $f \neq 0$. Выберем $\varepsilon>0$ настолько малым, чтобы $2 \eta<$ $\gamma-r(T)-\varepsilon$. Пусть $g \in C^{\infty}\left(\mathbb{C}^{n}\right)-$ ненулевая функция, удовлетворяющая условиям 1) и 2) леммы 4 при выбранном $\varepsilon$. Применяя оценку $(38)$ при $\alpha=r(T)+\varepsilon$, получаем

$$
\int_{E(R, \beta)}|g(z)| d m_{n}(z) \leqslant c_{1} \int_{E(R, \gamma)}|f(z)| d m_{n}(z),
$$

где постоянная $c_{1}>0$ не зависит от $R$. Отсюда и из (10) следует, что

$$
\int_{E(R, \beta)}\left|g^{p, q, l}(z)\right| d m_{n}(z) \leqslant c_{2} \int_{E(R, \gamma)}|f(z)| d m_{n}(z)
$$

при всех $p, q, l$ с постоянной $c_{2}>0$, не зависящей от $R$. Предположим, что $g^{p, q, l} \neq 0$. Тогда из свойства 1$)$ функции $g$ (см. лемму 4) и следствия 1 имеем

$$
\int_{E(R, \beta)}\left|\phi_{\lambda, p, q, l}(z)\right| d m_{n}(z) \leqslant c_{3} \int_{E(R, \gamma)}|f(z)| d m_{n}(z),
$$


где постоянная $c_{3}$ не зависит от $R$. Однако условия $(5),(6)$ и лемма 2 показывают, что последнее неравенство противоречиво для некоторой последовательности значений $R$, стремящейся к бесконечности. Это означает, что $g^{p, q, l}=0$ при всех $p, q, l$. Поэтому $g=0$ (см. [21, гл. 12 , теорема 12.2.3]), что противоречит выбору $g$. Таким образом, $f=0$, что и требовалось доказать.

ДоКАЗАТЕЛЬСТВО ТЕОРЕМЫ 2. Повторим рассуждения из доказательства теоремы 1 для случая, когда $E(R, \gamma) \subset \mathcal{O}$. Используя лемму 1 вместо следствия 1 и асимптотические формулы из леммы 2 , получим, что существует достаточно большое $R_{0}>0$, для которого $f=0$ в области $\left\{z \in \mathbb{C}^{n}:|z|>R_{0}\right\} \subset \mathcal{O}$. Поскольку $f \in \mathcal{D}_{T}^{\prime}(\mathcal{O})$, отсюда и из леммы 5 имеем утверждение теоремы.

ДОКАЗАТЕЛЬСТво ТЕОРЕмЫ 3. Рассмотрим функцию $f(z)=\phi_{\lambda, p, q, l}(z)$, где $\lambda \in \mathcal{Z}(\widetilde{T})$. Из следствия 1 получаем $\left(\mathfrak{L}-\lambda^{2} \mathrm{Id}\right) f=0$ в $\mathbb{C}^{n}$. Используя теорему о среднем для собственных функций оператора $\mathfrak{L}$ (см. [17, предложение 7]), находим

$$
f \star T=\widetilde{T}(\lambda) f=0 .
$$

Следовательно, $f \in \mathrm{RA}_{T}\left(\mathbb{C}^{n}\right)$. Кроме того, из $(20),(21)$ и леммы 2 вытекает, что $f$ удовлетворяет условию (7). Более того, если $N_{T} \neq \varnothing$, то при $\lambda \in N_{T}$ функция $f$ удовлетворяет (8). Таким образом, утверждения (i), (ii) теоремы 3 доказаны. Для доказательства утверждения (iii) достаточно повторить данные рассуждения для функции $f(z)=\psi_{\lambda, p, q, l}(z)$, где $\lambda \in \mathcal{Z}(\widetilde{T})$, с использованием леммы 2 .

\section{§ 7. Обобщения и следствия}

Теоремы 1, 2 допускают следующее обобщение.

Теорема 4. Пусть $\left\{T_{j}\right\}_{j=1}^{m}$ - семейство ненулевых распределений из $\mathcal{E}_{\natural}^{\prime}\left(\mathbb{C}^{n}\right)$, и пустъ $T=T_{1} \star \cdots \star T_{m}$. Предположим, что множество $\{1, \ldots, m\}$ является объединением попарно непересекающихся множеств $A_{1}, \ldots, A_{l}$ таких, что множества $\bigcup_{j \in A_{s}} \mathcal{Z}\left(\widetilde{T_{j}}\right), s=1, \ldots, l$, попарно не пересекаются. Тогда имеют место следующие утверждения:

(i) если $N_{T}=\varnothing, f_{j} \in \mathcal{D}_{T_{j}}^{\prime}\left(\mathbb{C}^{n}\right)$ при всех $j \in\{1, \ldots, m\}$,

$$
f=\sum_{j=1}^{m} f_{j} \in L^{1, \mathrm{loc}}\left(\mathbb{C}^{n}\right)
$$

и при некоторых $\gamma>r(T), \eta<\frac{1}{2}(\gamma-r(T))$ условие (5) выполнено для данных $f$ u $T$, то $\sum_{j \in A_{s}} f_{j}=0$ для всех $s \in\{1, \ldots, l\}$, аналогичный результат имеет место и при $N_{T} \neq \varnothing$, если вместо (5) выполнено (6);

(ii) если $r_{j} \geqslant 0, r=\max _{1 \leqslant j \leqslant m} r_{j}, G_{s}=\bigcap_{j \in A_{s}} \mathcal{B}_{r_{j},+\infty}, f_{j} \in \mathcal{D}_{T_{j}}^{\prime}\left(\mathcal{B}_{r_{j},+\infty}\right)$,

$$
f=\sum_{j=1}^{m} f_{j} \in L^{1, \mathrm{loc}}\left(\mathcal{B}_{r,+\infty}\right)
$$

и при некоторых $\gamma>r(T), \eta<\frac{1}{2}(\gamma-r(T))$ условие (6) выполнено для данных $f$ u $T$, mо $\sum_{j \in A_{s}} f_{j}=0$ в $G_{s}$ для всех $s \in\{1, \ldots, l\}$. 
ДоказАтельство. Из определений $f$ и $T$ следует, что $f \in \mathcal{D}_{T}^{\prime}\left(\mathbb{C}^{n}\right)$. В силу теоремы 1 это означает, что $f=0$. Используя лемму 3 , (iii), для $s \in\{1, \ldots, l\}$ определим распределения $U_{s}, V_{s} \in \mathcal{E}_{\natural}^{\prime}\left(\mathbb{C}^{n}\right)$ и $W_{s} \in \mathcal{D}^{\prime}\left(\mathbb{C}^{n}\right)$ по формулам

$$
\widetilde{U_{s}}=\prod_{j \in A_{s}} \widetilde{T_{j}}, \quad \widetilde{V_{s}}=\prod_{\substack{j=1 \\ j \notin A_{s}}}^{m} \widetilde{T_{j}}, \quad W_{s}=\sum_{j \in A_{s}} f_{j} .
$$

Поскольку $f=0$, имеем

$$
W_{s} \in\left(\mathcal{D}_{U_{s}}^{\prime} \cap \mathcal{D}_{V_{s}}^{\prime}\right)\left(\mathbb{C}^{n}\right) .
$$

Учитывая, что $\mathcal{Z}\left(\widetilde{U_{s}}\right) \cap \mathcal{Z}\left(\widetilde{V_{s}}\right)=\varnothing$, из [17, следствие 6$]$ получаем $W_{s}=0$. Таким образом, утверждение (i) доказано.

Для доказательства утверждения (ii) достаточно повторить рассуждения с использованием теоремы 2 вместо теоремы 1.

Нетрудно видеть, что без предположения о том, что множества

$$
\bigcup_{j \in A_{s}} \mathcal{Z}\left(\widetilde{T_{j}}\right), \quad s=1, \ldots, l,
$$

попарно не пересекаются, утверждения теоремы 4, вообще говоря, неверны (см. доказательство теоремы 3 в $\S 6$ ). Теорема 3 показывает также, что условия для роста $f$ в утверждениях (i) и (ii) теоремы 4 дают точную информацию о поведении главного члена в показателе экспоненты на бесконечности.

Рассмотрим теперь некоторые следствия теоремы 4 , усиливающие известную теорему о двух радиусах для искаженных сферических средних. Напомним, что искаженное сферическое среднее функции $f \in L^{1, \operatorname{loc}}\left(\mathbb{C}^{n}\right)$ для почти всех $z \in \mathbb{C}^{n}$ определяется равенством

$$
\left(f \star \mu_{r}\right)(z)=\int_{|w|=r} f(z-w) e^{\frac{i}{2} \operatorname{Im}\langle z, w\rangle_{\mathbb{C}}} d \mu_{r}(w),
$$

где $\mu_{r}$ - нормированная поверхностная мера на сфере $\mathbb{S}_{r}=\left\{w \in \mathbb{C}^{n}:|w|=r\right\}$. Известная теорема о двух радиусах утверждает, что если $f \in L^{1, \text { loc }}\left(\mathbb{C}^{n}\right)$ удовлетворяет условию

$$
f \star \mu_{r_{1}}=f \star \mu_{r_{2}}=0
$$

для некоторых фиксированных $r_{1}, r_{2}>0$ и система уравнений

$$
{ }_{1} F_{1}\left(a ; n ; \frac{r_{1}^{2}}{2}\right)=0, \quad{ }_{1} F_{1}\left(a ; n ; \frac{r_{2}^{2}}{2}\right)=0
$$

не имеет решения $a \in \mathbb{R}$, то $f=0$. Условие (40) для радиусов $r_{1}$ и $r_{2}$ является необходимым. Многочисленные результаты, связанные с теоремой о двух радиусах и ее аналогами, можно найти, например, в [7], [14, ч. 2], [17]. 
Обозначим через $K\left(r_{1}, r_{2}\right)$ множество решений $a \in \mathbb{R}$ системы (40). Пусть также $N(r)$ - множество нулей $\lambda$ функции

$$
{ }_{1} F_{1}\left(\frac{n-\lambda^{2}}{2} ; n ; \frac{r^{2}}{2}\right),
$$

удовлетворяющих условию (3). Положим $N\left(r_{1}, r_{2}\right)=N\left(r_{1}\right) \cup N\left(r_{2}\right)$.

СлеДСТВИЕ 2. Пусть $r_{1}, r_{2}>0 u K\left(r_{1}, r_{2}\right)=\varnothing$. Тогда выполнены следующие утверждения:

(i) если $f_{1}, f_{2} \in L^{1, \text { loc }}\left(\mathbb{C}^{n}\right)$,

$$
f_{1} \star \mu_{r_{1}}=f_{2} \star \mu_{r_{2}}=0 \quad \text { в } \quad \mathbb{C}^{n},
$$

кроме того, $N\left(r_{1}, r_{2}\right)=\varnothing$ и функиия $f=f_{1}-f_{2}$ удовлетворяет условию (5) при некоторых $\gamma>r_{1}+r_{2}, \eta<\frac{1}{2}\left(\gamma-r_{1}-r_{2}\right)$, то $f_{1}=f_{2}=0$, аналогичный результат имеет место и при $N\left(r_{1}, r_{2}\right) \neq \varnothing$, если вместо (5) выполнено (6);

(ii) если $R \geqslant 0, f_{1}, f_{2} \in L^{1, \text { loc }}\left(\mathcal{B}_{R,+\infty}\right)$,

$$
f_{j} \star \mu_{r_{j}}=0 \quad \text { в } \quad \mathcal{B}_{R+r_{j},+\infty}, \quad j=1,2,
$$

и функция $f=f_{1}-f_{2}$ удовлетворяет (6) при некоторых $\gamma>r_{1}+r_{2}, \eta<$ $\frac{1}{2}\left(\gamma-r_{1}-r_{2}\right)$, mo $f_{1}=f_{2}=0$.

ДокАЗАТЕЛЬство следует из теоремы 4 , если положить в ней $m=2$, $T_{1}=\mu_{r_{1}}, T_{2}=\mu_{r_{2}}$. Отметим также, что при $K\left(r_{1}, r_{2}\right) \neq \varnothing$ из условий следствия 2 и теоремы 4 вытекает, что $f_{1}=f_{2}$. Как уже отмечалось, в этом случае существуют ненулевые $f_{1}, f_{2}$, удовлетворяющие (41) (см., например, доказательство теоремы 3).

Следующий результат касается случаев, когда условие (39) для одного из радиусов можно заменить оценкой сверху искаженного сферического среднего, но, тем не менее, утверждение теоремы о двух радиусах сохраняется.

СлеДСТВИЕ 3. Пусть $r_{1}, r_{2}>0 u K\left(r_{1}, r_{2}\right)=\varnothing$. Тогда выполнены следующие утверждения:

(i) если $N\left(r_{1}\right)=\varnothing, g \in L^{1, \text { loc }}\left(\mathbb{C}^{n}\right), g \star \mu_{r_{1}}=0$ в $\mathbb{C}^{n}$ и функиия $f(z)=$ $\left(g \star \mu_{r_{2}}\right)(z)$ удовлетворяет условию (5) при некоторьх $\gamma>r_{1}, \eta<\frac{1}{2}\left(\gamma-r_{1}\right)$, то $g=0$, аналогичный результат имеет место и при $N\left(r_{1}\right) \neq \varnothing$, если вместо (5) выполнено (6);

(ii) если $R \geqslant 0, g \in L^{1, \operatorname{loc}}\left(\mathcal{B}_{R,+\infty}\right), g \star \mu_{r_{1}}=0$ в $\mathcal{B}_{R+r_{1},+\infty}$ и функиия $f(z)=$ $\left(g \star \mu_{r_{2}}\right)(z)$ удовлетворяет условию (6) при некоторых $\gamma>r_{1}, \eta<\frac{1}{2}\left(\gamma-r_{1}\right)$, $\operatorname{mog} g=0$.

ДокАзАтельство. Докажем утверждение (ii) (утверждение (i) доказывается аналогично). Из условия следует, что $f \star \mu_{r_{1}}=0$ в $\mathcal{B}_{R+r_{1}+r_{2},+\infty}$. Тогда по теореме 2 имеем $f=0$ в $\mathcal{B}_{R+r_{2},+\infty}$. Это означает, что $g \star \mu_{r_{2}}=0$ в $\mathcal{B}_{R+r_{2},+\infty}$. Полагая $f_{1}=g, f_{2}=-g, T_{1}=\mu_{r_{1}}, T_{2}=\mu_{r_{2}}$, из теоремы 4 получаем требуемое утверждение. 


\section{Список литературы}

1. G. B. Folland, Harmonic analysis in phase space, Ann. of Math. Stud., 122, Princeton Univ. Press, Princeton, NJ, 1989.

2. S. Thangavelu, Lectures on Hermite and Laguerre expansions, Math. Notes, 42, Princeton Univ. Press, Princeton, NJ, 1993.

3. C. Berenstein, D.-Ch. Chang, J. Tie, Laguerre calculus and its applications on the Heisenberg group, AMS/IP Stud. Adv. Math., 22, Amer. Math. Soc., Providence, RI, 2001.

4. Ф. Йон, Плоские волны и сферические средние в применении к дифференциальным уравнениям с частными производными, ИЛ, М., 1958; пер. с англ.: F. John, Plane waves and spherical means applied to partial differential equations, Interscience Publ., New York-London, 1955.

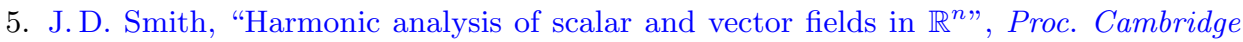
Philos. Soc., 72:3 (1972), 403-416.

6. A. Sitaram, "Fourier analysis and determining sets for Radon measures on $\mathbb{R}^{n}$ ", Illinois J. Math., 28:2 (1984), 339-347.

7. S. Thangavelu, "Spherical means and CR functions on the Heisenberg group", J. Anal. Math., 63:1 (1994), 255-286.

8. R. Rawat, A. Sitaram, "Injectivity sets for spherical means on $\mathbb{R}^{n}$ and on symmetric spaces", J. Fourier Anal. Appl., 6:3 (2000), 343-348.

9. L. Flatto, "The converse of Gauss's theorem for harmonic functions", J. Differential Equations, 1:4 (1965), 483-490.

10. О.А. Очаковская, "О функциях с нулевыми интегралами по шарам фиксированного радиуса на полупространстве”, Докл. РАН, 381:6 (2001), 745-747; англ. пер.: O. A. Ochakovskaya, "On functions with zero integrals over balls of fixed radius on a half-space", Dokl. Math., 64:3 (2001), 413-415.

11. О.А. Очаковская, "Теоремы типа Лиувилля для функций с нулевыми интегралами по шарам фиксированного радиуса", Докл. РАН, 415:2 (2007), 171-173; англ. пер.: O. A. Ochakovskaya, "Liouville-type theorems for functions with zero integrals over balls of fixed radius", Dokl. Math., 76:1 (2007), 530-532.

12. О.А. Очаковская, "Точные характеристики допустимой скорости убывания ненулевой функции с нулевыми шаровыми средними", Матем. сб., 199:1 (2008), 47-66; англ. пер.: О. A. Ochakovskaya, "Precise characterizations of admissible rate of decrease of a non-trivial function with zero ball means", Sb. Math., 199:1 (2008), $45-65$.

13. О.А. Очаковская, "Мажоранты функций с нулевыми интегралами по шарам фиксированного радиуса", Докл. РАН, 420:5 (2008), 598-600; англ. пер.: O. A. Ochakovskaya, "Majorants of functions with zero integrals over balls of fixed radius", Dokl. Math., 77:3 (2008), 446-448.

14. V.V. Volchkov, Integral geometry and convolution equations, Kluwer Acad. Publ., Dordrecht, 2003.

15. S. Thangavelu, "Mean periodic functions on phase space and the Pompeiu problem with a twist", Ann. Inst. Fourier (Grenoble), 45:4 (1995), 1007-1035.

16. Г. Бейтмен, А. Эрдейи, Высиие трансцендентные функции, т. 1, 2, Наука, М., 1973-1974; пер. с англ.: A. Erdélyi, W. Magnus, F. Oberhettinger, F. G. Tricomi, Higher transcendental functions, vols. I, II, McGraw-Hill, New York-Toronto-London, 1953.

17. В. В. Волчков, Вит. В. Волчков, "Уравнения свертки на многомерных областях и редуцированной группе Гейзенберга", Матем. сб., 199:8 (2008), 29-60; англ. пер.: V. V. Volchkov, V. V. Volchkov, "Convolution equations in many-dimensional domains and on the Heisenberg reduced group", Sb. Math., 199:8 (2008), 1139-1168. 
18. M. Shahshahani, A. Sitaram, "The Pompeiu problem in exterior domains in symmetric spaces", Integral geometry (Brunswick, Maine, 1984), Contemp. Math., 63, Amer. Math. Soc., Providence, RI, 1987, 267-277.

19. В. В. Волчков, "Теоремы о шаровых средних на симметрических пространствах", Maтем. сб., 192:9 (2001), 17-38; англ. пер.: V.V. Volchkov, "Theorems on ball mean values in symmetric spaces", Sb. Math., 192:9 (2001), 1275-1296.

20. М. А. Евграфов, Асимптотические оценки и целье функиии, 3-е изд., Наука, М., 1979; англ. пер. 1-го изд.: М. A. Evgrafov, Asymptotic estimates and entire functions, Gordon and Breach, New York, 1961.

21. У. Рудин, Теория функиий в единичном шаре из $\mathbb{C}^{n}$, Мир, М., 1984; пер. с англ.: W. Rudin, Function theory in the unit ball of $\mathbb{C}^{n}$, Grundlehren Math. Wiss., 241, Springer-Verlag, New York-Heidelberg-Berlin, 1980.

В. В. Волчков (V. V. Volchкоv)

Донецкий национальный университет, Украина

Поступило в редакцию

E-mail: valeriyvolchkov@gmail.com

12.10 .2010

Вит. В. Волчков (Viт. V. Volchкоv)

Донецкий национальный университет, Украина

E-mail: v.volchkov@mail.donnu.edu.ua 